\title{
A Novel Framework for Predicting and Managing Comorbid Diseases using Neutrosophic Logic and Machine Learning
}

\author{
A. E. Okpako \\ Department of Mathematical Sciences \\ Edwin Clark University, Kiagbodo \\ Delta State, Nigeria
}

\author{
R. E. Ako \\ Department of Mathematical Sciences \\ Edwin Clark University, Kiagbodo \\ Delta State, Nigeria
}

\begin{abstract}
Emerging realities in medical research had advocated for a shift from single disease diagnosis mostly in clinical diagnosis of geriatric patients since most geriatric patients presents more than one disease complications. Comorbidity is visibly a phenomenon predominantly seen in elderly patients thus has made the management of diseases in such patients' complex. A metric is needed which would be an indispensable tool for prioritizing treatment or developing clinical guidelines so as to handle the blizzards of risks of overtreatment and inappropriate prescription. This metric would provide an evidence about how both conditions and care processes interact and as such would assist and/or complement human practitioners mostly in areas where there are only General Practitioners to handle comorbid disease diagnosis, treatment and management. In this paper, we present a framework using Artificial Neural Network whose inference mechanism is driven by Neutrosophic logic, all being mechanism employed in soft computing so as to ensure intelligent capability in handling comorbidity. This framework is generic and could be used for any comorbid disease of interest.
\end{abstract}

\section{Keywords}

Comorbidity, Genetic Programming, Neutrosophic Logic, Comorbidity burden, Poly-pharmacy, Pathology, Clinical Practice Guidelines, Clinical Pathway, Advanced Drug Reaction, pharmacogenomics.

\section{INTRODUCTION}

There is a continual and consistent penetration of computers and its related devices into every area of human endeavors. Central to this, is the drive by researchers to make computing devices mimic and reason like human beings which is best expressed as soft computing. Artificial Intelligence like a mother-hen has given birth to many concepts such as pattern recognition, machine learning, Artificial Neural Networks, Genetic Programming, Natural Language Processing, Expert Systems just to mention a few. One cannot delve into the concept of Artificial Intelligence and its application without mentioning the pioneering work of John MCcarthy who has been unanimously acclaimed father of Artificial Intelligence since he coined the word. Professor Edward Feigenbaum of Standford University defined Expert System as the use of knowledge and inference procedures to solve problems that are difficult enough to require significant human expertise for their solution. The use of computers armed with these Artificial intelligence sub-concepts have been used superlatively in efficiently and seamlessly managing patient data in medical institutions through clinical decision support systems. There are varied definitions of decision support systems in literature but central to them all is the ability to help users to make proper decisions mostly in dicey situations.
Power (1999) defined decision support system (CDS) as an interactive computer based system intended to help users or decision makers utilize data and models in order to identify and solve problems and make decision. In line with this, clinical decision support roadmap project opine that CDS is providing users with knowledge and person specific or population information, intelligently filtered or presented at appropriate times, to foster better health processes, better individual patient care, and better population health. Chen and Lee (2002) also define clinical decision support system as an active knowledge base where two or more items of patients data are used to generate case specific recommendation, in other words it makes use of knowledge management to achieve clinical advice for patient care on some number of items of patients data.

The management of diseases and patient in Nigeria health care services is gradually becoming difficult in elderly patient due to instances of co-occurrence of two or more diseases in one patient. This complexity arises from the fact that there could be interplay of pathology in the two diseases, drug interactions between them in time of treating an index disease and such adequate treatment plan should be put in place to address contradictory or redundant recommendations or treatment. Elderly patients have been shown to develop two or more chronic disease as the age of the patient's increases, Van den Akker et al (1998) opine that older adults generally have multiple medical problems, and no single medical issue can be evaluated and treated in isolation. This situation does not start immediately rather through progression of an index disease either through interaction of drugs in the body system which was meant for the treatment of the index disease or other clinical factors. These situations where two or more diseases can coexist in the same patient have been called many names in medical literature such as comorbidity, multi-morbidity, poly-pharmacy just to mention a few, but we are confine to use comorbidity in our research. Comorbidity is a multidimensional or hydra headed concept in medical diagnosis and in disease management because societal/institution as well as individual patients has a contributory factor to its emergence in the first place. Its management has shown an obvious concern, since a proper diagnosis of comorbid disease is a panacea to a good health service delivery to elderly patients which would in turn lead to increased life expectancy of the geriatric patients.

Comorbidity is not static rather it is dynamic such that the issue of which of the diseases is the index disease (could only be ascertained either by time/sequence or interaction between the two diseases), the treatment interaction in terms of drugs, the current level of function of the patient ascertained through necessary biomarkers or signs, as well as the accumulated effect of the dysfunction of the past should be considered in a 
bid to design a system that could help in the management of comorbidity. According to Ammin et al (2013), medical errors are costly and gravely harmful and as such needs timely intervention to curb. In light of this, it is imperative to have a clinical decision support system that can assist in the management of comorbidity mostly in elderly patients.

\section{CLINICAL DIAGNOSIS}

Oguntimelehin et al [26] opined that medical diagnosis is simply the task of categorization which allows physician to make predictions using clinical situations and to determine appropriate cause of action. They said it is a complex decision making process that involves a lot of vagueness and uncertainty management especially when the disease has multiple symptoms. Diagnosis has been seen generally as the identification of the nature and cause of a certain phenomenon. Several disciplines make use of it but we are only considering it in the parlance of medical science and to put it in more simplistic form, it is the answer to the question of whether a system( in this case human body) is malfunctioning or not, and to the process of computing the answer. Expert diagnosis would not be trivialized in this regard, which is majorly based on experience with the system. Using this experience, a mapping is built that efficiently associates the observations to the corresponding diagnoses.

\subsection{Comorbidity}

Medical research has been saturated with single disease diagnosis but issue of two or more diseases simultaneously presented by a single patient has drawn researcher's attention in recent time; its adverse effects has affected the proper diagnosis and management of diseases in such situations. It is an important issue in medical science not only due to adverse effect it posits in terms of complex and confusing diagnostic classification, overlapping clinical manifestation and pathogenesis, systemic disease concepts but also in the context of proper and appropriate pharmacotherapy, selfmedication and health care utilization as well as drug development strategy. Comorbidity has been referred to with different names and has been seen from different angles and as such it has presented itself with varied meanings and synonyms in medical literature. The coexistence of two or more diseases in patients is not by chance as there are several factors responsible for it such as societal, patient centered (current status and the past dysfunction), age, etc. Feinstein (1970) was the first to observe this phenomenon and he coined the word comorbidity which he used to denote the coexistence of two or more diseases, pathological conditions or clinical entities in the same patient. Feinstein went further to state that the definition includes any clinically relevant phenomenon separate from the primary disease of interest that occurs while the patient is suffering from the primary disease, even if this secondary phenomenon does not qualify as a disease per se.

Jakovljevic and Ostojic (2013) opine that that there are three broad meanings of comorbidity outline herewith:

i. two or more medical conditions existing simultaneously but independently with each other

ii. two or more medical conditions existing simultaneously and interdependently with each other

iii. two or more medical conditions existing simultaneously regardless of the causal relationship

Valderas et al (2009) opine that comorbidity is the presence of additional disease in relation to an index disease. Starfield
(2006) opine that comorbidity is a term that refers to the simultaneous presence of multiple health conditions when there is an index condition and other unrelated conditions. Comorbidity comes in different flavors and shapes such as etiological, non-etiological, concordant and discordant, organic and non-organic, concurrent and successive, transsyndromal and trans-nosological, unidirectional and bidirectional just to mention a few.

According to Fraccarro et al (2015), comorbid diseases are poised with the following problems outline herewith.

i. Interaction between pathologies

ii. Duplication of tests- The increased degree (the number of disease) of comorbidity would necessitate an equal increase in the number of experts and as such would bring about excessive number of communication interfaces between the experts and the patients which ultimately leads to duplication of test and harmful decision made on the basis of uncertain information.

iii. Difficulties in adhering to often conflicting clinical practice guidelines

iv. Obstacle in continuity of care

v. Confusing self-management information

vi. Medical errors- according to Ammin et al (2013) medical errors are both costly and harmful. They opine that it cause thousands of death worldwide.

\subsection{Poly-Pharmacy}

One of the major considerations in managing comorbid patients is the issue of poly-pharmacy. Poly-pharmacy is seen as the use of multiple medications or the use of a medication that is not indicated. Fulton and Allen (2005) opine that polypharmacy is the prescription and use of multiple drugs to deal with concomitant multiple diseases. Von Lueder and Atar (2014) opine that poly-pharmacy is an interaction between specific drugs to treat comorbid patients and as such augments the complexity of management of comorbidity. They observed that in most cases, comorbidity often faces the dilemma of mutually exclusive drugs, that is, drugs that improve one condition and deteriorate another and vice versa. For example, in Heart Failure and comorbid respiratory disease, b-blockers represent a cornerstone of contemporary Heart Failure pharmacotherapy but may have an adverse impact on pulmonary function. Von Lueder and Atar (2014) also opine that an estimate of the individual contributions of comorbid situation to current clinical status facilitates balancing drug therapies. A metric is needed which would be indispensable tool for prioritizing treatment or developing clinical guidelines so as to handle the blizzards of risks of overtreatment and inappropriate prescription.

\section{ANALYSIS OF THE EXISTING AND RELATED FRAMEWORKS}

Proper diagnosis and management of geriatric patients as regards comorbid disease situations should be done in order to minimize or alleviate the mismanagement or misdiagnosis in such situations. Many existing system have employed different approaches in ameliorating the effect of comorbidity diagnosis yet there is need for improvement since a good and reliable system would help in the proper and appropriate management of geriatric patients mostly in Nigeria; considering the fact that we have just a handful of experts in their respective domains or fields. Three frameworks was considered here, they are Comorbidity Ontological Modeling and Execution (COMET) by Abidi et al(2010) , Perinatal 
Decision Support system(PEDSS) by Gunaratman et al (2013), and Comorbidity Ontological Framework for Intelligent Prediction (COFIP) by Unuiri et al(2014). A detailed review and analysis of existing system was carried out in order to bring to fore areas to improve on in order to manage comorbidity in elderly patients. We reviewed the following:-

i. The approaches and methods used in the existing system in knowledge construction

ii. The inference mechanism in handling confusability due to comorbidity

iii. Support for extent of comorbidity.

However our focus is on the latest framework which is Unuiri et al (2014) COFIP framework.

\subsection{Comorbidity Ontological Framework for Intelligent Prediction}

This is an ontological based decision support framework for handling comorbidity by aligning of ontological modeled Clinical Practice Guideline and Clinical Pathways. It used neural network and decision trees, which ensure intelligibility in managing cases of comorbidity of Cataract and Glaucoma, though it is adaptable for other chronic disease of interest. The architecture of the system or COFIP framework is shown in Figure 1.

\subsection{Limitation of the Existing System}

Though the existing system would able to perform the classification and ultimate prediction of the tendency of diseases to degenerate into comorbid situation, however, there are some salient points that need improvement for optimal prediction and use in managing comorbidity.

The under listed are the drawbacks of the existing systems:

i. One can only predict the presence or absence of precondition $\mathrm{A}$ and $\mathrm{B}$ but not the degree of membership of the disease to either precondition A or precondition B. Thus, an estimate of individual contributions of the comorbid diseases to the current comorbid situation is not there which could facilitates balancing drug therapies.

ii. The degree of comorbidity is missing. Knowing the extent of the comorbidity would help in the drug prescription (dose and frequency or identify ADR) since comorbid patients are prone to polypharmacy.

iii. Though uncertainty of other forms was handled but uncertainty as a result of indeterminacy was not handled. In some single disease diagnosis, outlier detection has been applied to the training instances or the desired dataset but not on the testing datasets or the input features that is not handy which might contain outliers too. Also, with unnecessary variables adding noise and complexity to the problem, it reduces the likelihood of identifying positive cases. Sometimes as a result of drug interaction, it can affect the values of symptoms of the index disease or the secondary disease thus having incomplete and inconsistent values.

iv. The features were taken into the Artificial Neural Network directly without an adequate feature selection process, thereby there is over reliance on the knowledge representation and preprocessing stage. This could lead to over-fitting in the Neural
Network that could affect its power of generalization. The over-fitting problem was handled in the decision tree using pruning but not in the Artificial Neural network. Bermingham et al (2013) observed that feature selection helps in enhancing generalization by reducing over fitting.

\section{DESIGN OF THE PROPOSED FRAMEWORK}

Several researchers have applied different approaches to diagnosing comorbid diseases and from the analysis of our considered existing system, several limitations or drawbacks were observed and highlighted for our proposed framework to tackle. In this work, the key point is to employ an ensemble Neural network system made up of the three preconditions in order to get the degree of membership in precondition A, precondition $\mathrm{B}$ and comorbid situation and their associated confusability measurements which gives a tripartite membership of neutrosophic logic which would ultimately be used to make decisions in terms of drug prescription or further clinical examination. The burden of comorbidity captured in the network is aimed at measuring the stochastic acquisition error and indeterministic-related problems in lieu of having a better way of managing poly-pharmacy in comorbidity. It should be noted that a proper pharmacotherapy is a panacea to proper management of comorbid patients.

Our proposed system exhibit three major characteristics or uniqueness;

i. Three classes of disease: The consideration of three classed neural networks ensemble, of which the precondition $\mathrm{A}$, precondition $\mathrm{B}$ and comorbid precondition $\mathrm{C}$ corresponds to each class separately so as to know the extent of contribution of each disease to the current comorbid patient which would enable practitioners to balance dug therapy.

ii. The introduction of degree of confusability for the prediction of the correct classes rather than the black box output of the neural networks; this would ultimately lead to better performance in terms of sensitivity and specificity.

iii. The inference mechanism of using the notion of neutrosophic logic -having a tripartite membership rather than comparing the output of ANN and Decision tree for decision making process. Neutrosophic logic up till now has a superlative representation and capability of handling uncertainty mostly in situations that have indeterminacy when compared to fuzzy logic and other logics because it looks at the reliability of the information which is in line with support theory in clinical diagnosis.

iv. According to William Osler, variability is the law of life and as no two faces are the same, so no two bodies are alike and no two individuals responds alike and behave alike under abnormal circumstances which we know as disease. Likewise comorbidity may not present itself the same in all patients. This variability and its pattern were captured in the framework expressed as confusability measurement or indeterminacy.

The existing framework or architecture is shown in Fig. 1 while the proposed architecture is shown in Figure 2 and 3 


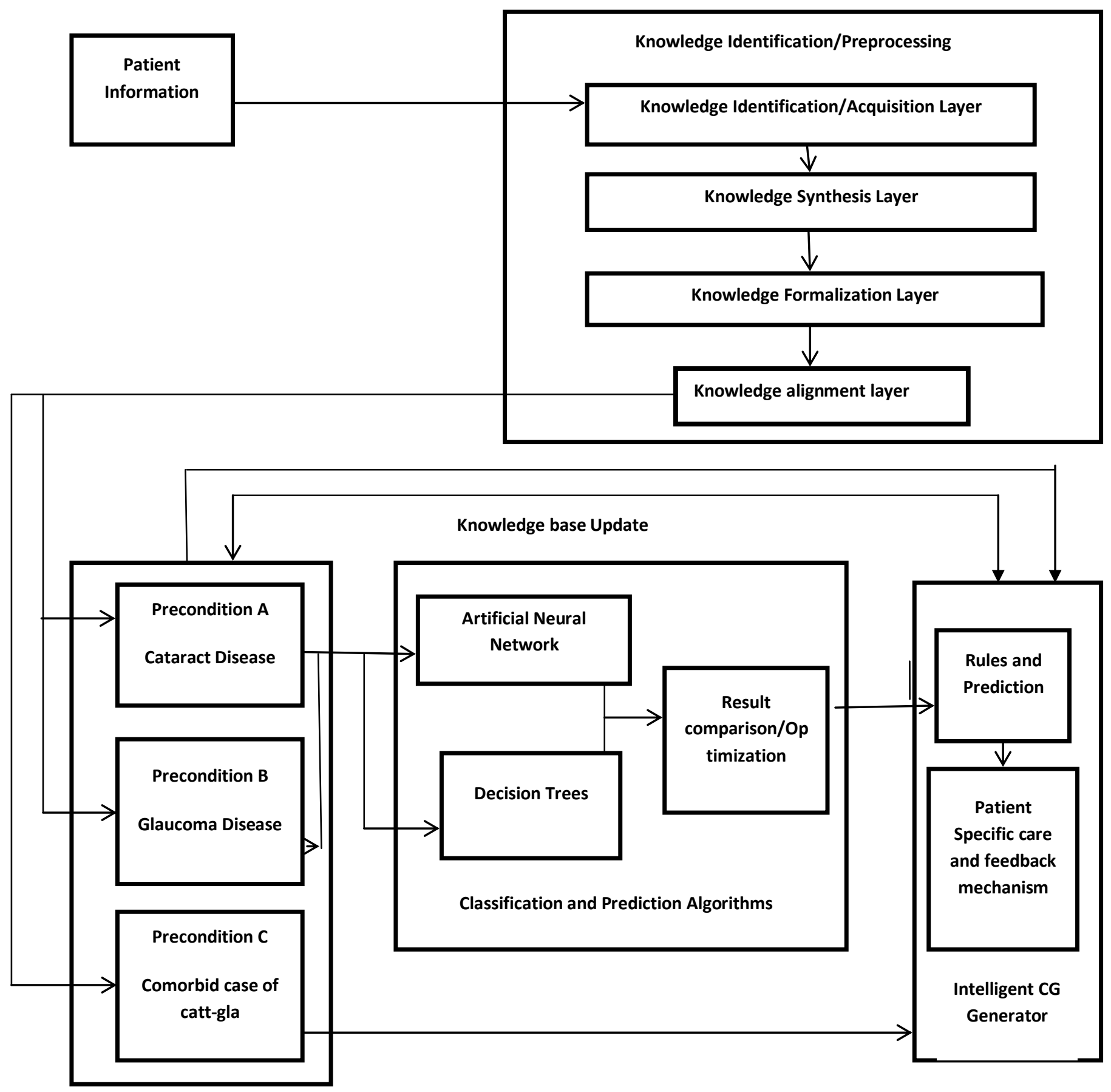

Figure 1: COFIP Framework (Source: Unuiri et al(2014).) 


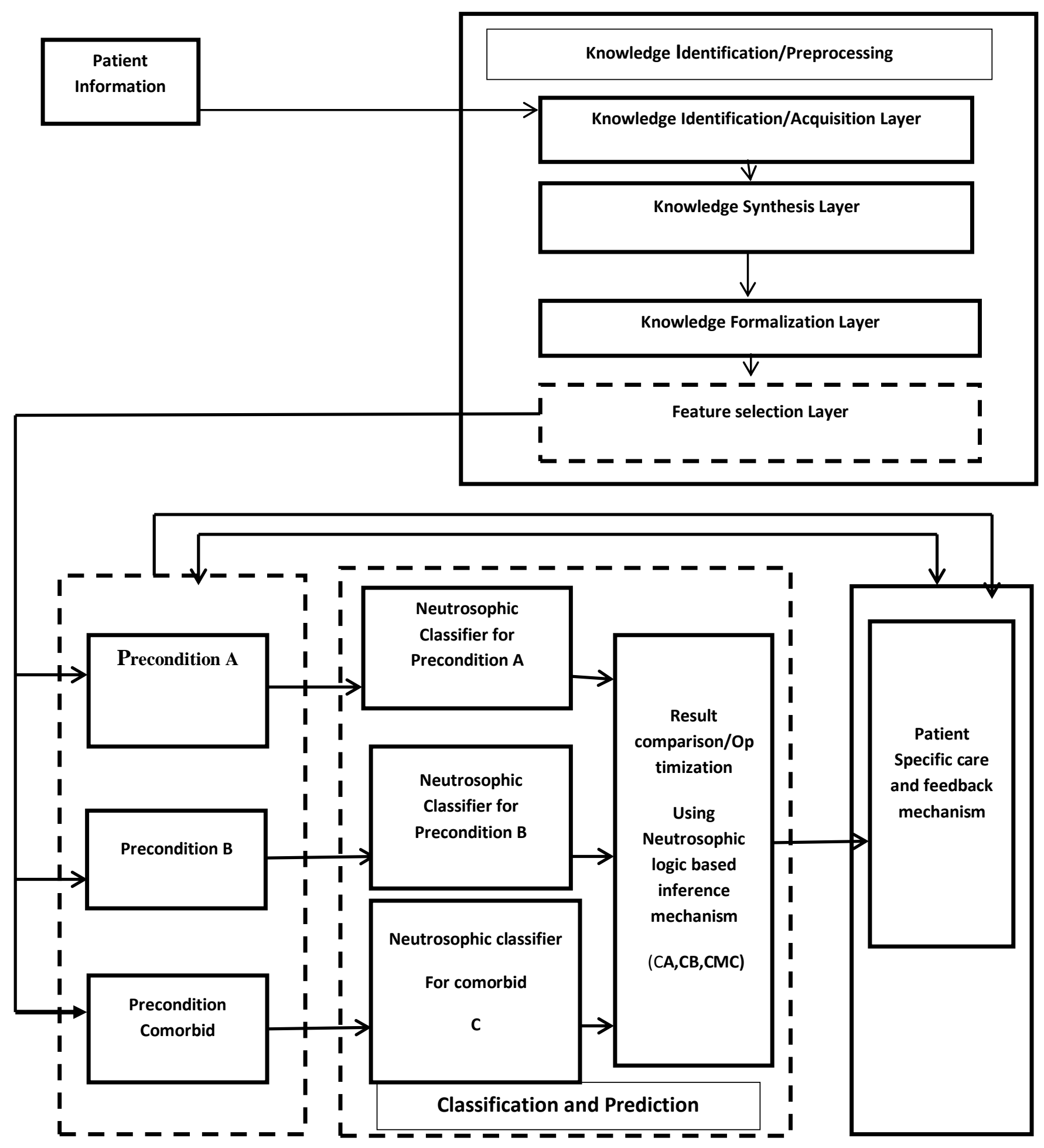

Fig. 2: Proposed system architecture 


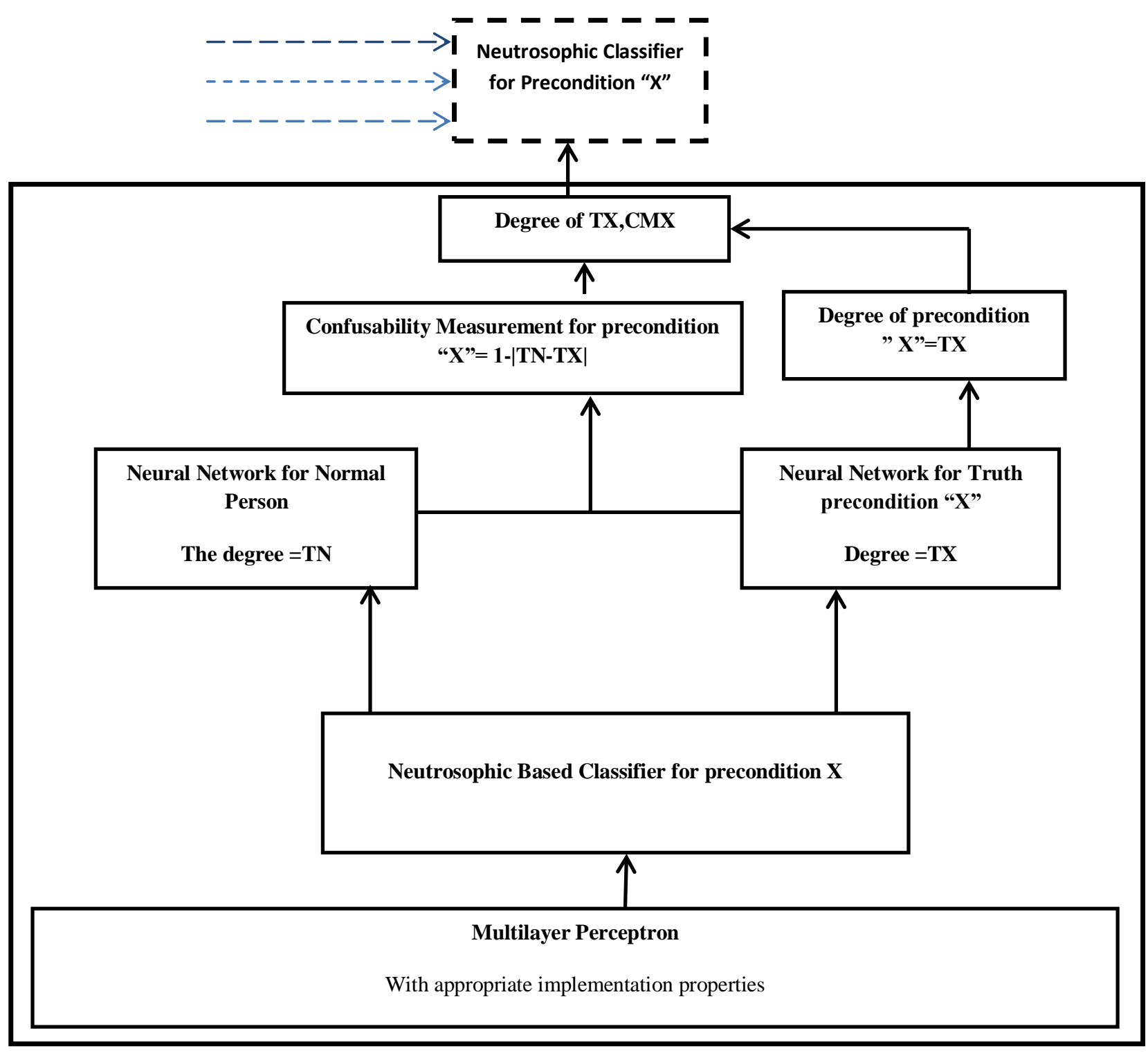

Figure 3: Expanded subcomponent for classification and prediction

\subsection{Underlying Concepts of the Proposed System}

This section talks about the underlying concepts that make up the proposed framework

\section{a) Feature Selection / Inputs}

Feature selection or variable selection is an indispensable stage in any patterned diagnosis as it related to machine learning. The appropriate features are selected by a feature selector, because a lot depends on it for the entire system to be at its best in its classification performance even though using the best classifier hence the selector should reduce dimension of pattern vector to a lower as well as cover useful information of the original vector. It is the reduced feature vectors that are ultimately given to the classifier for the classification. The appropriate features designated as precondition A, Precondition B and Precondition $\mathrm{C}$ are described as :
Precondition A- This contains all relevant data/features for the presented disease (Precondition A only).

Precondition B- This contains all relevant data/features for presented disease (Precondition B only).

Precondition C- This contains all information about a patient with data from both precondition A and B simultaneously.

Precondition Normal- This contains all relevant attribute for a person with neither preconditions.

The features of the different preconditions are fed into their respective neutrosophic classifiers.

b) Artificial Neural Network

Neural network is a supervised machine learning algorithm has been chosen owing to its superlative ability in handling nonlinear datasets and its effectiveness in modeling biological data. It is infused with neutrosophic indetermistic measurement for better classification towards handling any 
form of uncertainty and indeterminism in lieu of improving the performance of the neural network in diagnosing and managing comorbid diseases. It has been used extensively in clinical decision support system either alone or hybridized with others algorithms. The neural networks are separated for each of the preconditions from whence corresponding confusability measurement is calculated. Different neural network properties are chosen for each of the preconditions in line with the performance of various preconditions. In other words, precondition A might not have the same neural network properties with precondition $B$ and vice versa.

\section{c) Fuzzy logic/ Fuzzification of inputs}

It is a known fact that scientists tend to look for typical, normal phenomena while medical sciences look out for the atypical, abnormal, morbid phenomena. The pertinent question to ask is "how does one find a law or a relation for these irregular phenomena". This is the central problem in medical domain. In the same vein one would also ask what relation or function should be adopted between them in order to obtain a rational understanding? It is on this note that one tries to look for the abnormal, irregular, atypical phenomenon in order to characterize such situation. Ludwik Fleck (18961961), a polish philosopher and physician was the first to notice this and rightly presented it in his book titled "Genesis and Development of a scientific fact" in 1926. He opined that there are some specific features of the medical way of thinking. He also went further to state that there was no sharp border between these phenomena. Smooth transitions in the space of diseases feed from one phenomenon and very small variations could be the reasons that the physician diagnoses a patient's with $\mathrm{x}$ instead of $\mathrm{y}$. It is an established conception that physician tends to rely on experience and intuition and not on rational and strong rules to deduce from the patients' data to a disease.

Fuzzy logic proposed by Zadeh which deals with notion of degree of belongingness in which value ranges between 0 and 1 or true or false respectively. The degree is expressed as the membership function of a mapping from a set A to the interval $[0,1]$ using a linguistic variable. Membership function denoted by $A_{s}(x)$ represents a unique fuzzy set $S$. This logic is quite different from previous logic theory because rather than considering the categorical boundaries of 1 and 0 only for a classes, it seeks for the degree of membership into the respective classes or sets. If the value is 0 , it means that the element does not belong to the fuzzy set $S$, otherwise 1 , which means that it belongs fully to the fuzzy set S.

This is best captured in equation 1

$$
\mathbf{A}_{\mathbf{s}}(\mathbf{x}): \longrightarrow \mathbf{X}[\mathbf{0},] \text {. }
$$

Zadeh (1999) stated that "In a given pathology, which we denote by $\mathbf{S}$ a set of symptoms, $\mathbf{D}$ a set of diagnoses and $\mathbf{P}$ a set of patients. What we call medical knowledge is a fuzzy relation, generally denoted by $\mathbf{R}$, from $\mathbf{S}$ to $\mathbf{D}$ expressing associations between symptoms or syndromes, and diagnoses or group of diagnoses".

\section{d) Neutrosophic Logic}

Neutrosophic Logic represents an alternative to the existing logics as a mathematical model of uncertainty, vagueness, ambiguity, imprecision, undefined, unknown, incompleteness, inconsistency, redundancy, contradiction as opine by Smarandache (1999). It is a non-classical logic. It is a logic in which each proposition is estimated to have the percentage of truth in a subset $\mathrm{T}$, the percentage of indeterminacy in a subset
I, and the percentage of falsity in a subset F, where T, I, F are defined above, is called Neutrosophic Logic.

A neutrosophic set $A$ in $X$ is characterized by a truth membership function $T A$, an indeterminacy- membership function $I A$ and a falsity-membership function $F A$. $T A(x)$, $I A(x)$ and $F A(x)$ are real standard or non-standard subsets of

]$-0,1+[$.

That is

TA: $\mathrm{X} \varepsilon]-0,1+[$

IA: $X \varepsilon]-0,1+[$

FA: $X \varepsilon]-0,1+[$

There is no restriction on the sum of $T A(x), \operatorname{IA}(x)$ and $F A(x)$, so

$-0 \leq \operatorname{supTA}(x)+\operatorname{supIA}(x)+\operatorname{supFA}(x) \leq 3+\ldots \ldots \ldots \ldots \ldots . .2$

The notion of neutrosophic logics paints a better picture owing to the 3-dimensional definition of comorbidity by Jakovljevic and Ostojic (2013) which clearly aligns with the neutrosophic notions of interdependency and independency of events as opined by Smarandache (2006). The alignment is shown herewith:

i. Two or more medical conditions existing simultaneously but independently with each otherIndependent Events-Degree of precondition A+ Degree of precondition $B>1$

ii. Two or more medical conditions existing simultaneously and interdependently with each other.- Interdependent Events-Degree of Precondition A+ Degree of Precondition B $<1$

iii. Two or more medical conditions existing simultaneously regardless of the causal relationship

\section{e) Result Comparison and Inference Mechanism}

In this framework, neutrosophic-based inference technique is used which not only classifies the object as the class in the conclusion part of the prediction using the under-listed equations but also tells the practitioner the kind of relationship that exists between the two diseases shown to be comorbid as the case may be.

If the degree of comorbid precondition is high, that means there is high risk of advanced drug reaction, thus the practitioner has to look at the drug prescription for that comorbid case so as offer a solution to the advance drug reaction.

If a prediction is concluded to be comorbid-precondition $\mathrm{C}$, you can then prioritize treatment as follows:

If $(\mathrm{CA}<\mathrm{CB})$

Prioritize Precondition A:

\}

Else

\section{\{}

Prioritize Precondition B

\}

Where CA and CB denotes Confusability Measurement for Precondition $\mathbf{A}$ and Precondition $\mathbf{B}$ respectively. It is based on the tripartite membership of these components; a decision of triage of treatment is appropriately made. 


\section{CONCLUSION}

A proper and appropriate management of comorbidity mostly in geriatric patients is a panacea for reduction in frequency of hospitalization and ultimately reduction in death cases as a result of mismanagement. Other frameworks have been used and adopted in the past, however poly-pharmacy was considered in our design since the extent of the individual disease contribution to such current status of comorbidity would enable a practitioner to balance the drug therapy or would help the practitioner to know what disease to prioritize in the development of clinical guidelines. In quantifying the extent of comorbidity, three class Neural Network is used to predict the degree of presence of precondition A, Normal person in absence of both disease conditions, Precondition B and Precondition $\mathrm{C}$. The vagueness of each of the conditions are calculated designated as CA,CM, and CB. The inference mechanism is also improved by employing the concept of neutrosophic logic thereby having a tripartite membership rather than just classification exercise of presence or absence of a certain precondition in order to make therapeutic recommendations. With these consideration, it is hoped that there is going to be an obvious improvement in the system performance in terms of handling and managing comorbid patients and eventually renders a proper diagnosis situation and ultimately being used in the decision making process.

Future work will delve into the implementation procedure of the framework for the diagnosis and management of comorbid diseases using two comorbid diseases and the result from the implementation and evaluation will be provided.

\section{REFERENCES}

[1] Abidi S.R. (2010). A Knowledge Management Framework to Develop, Model, Align and Operationalize Clinical Pathways to Provide Decision Support for Comorbid Diseases. http://dalspace.library.dal.ca/handle/ 10222/13009.

[2] Bressler R. and Bahal J.J (2003). Principles of drug therapy for elderly patients. Mayo Clin Proc; 78(12):1564-77.

[3] Chen, J.Q and Lee, S.M. (2002). An exploratory cognitive DSS for strategy decision making. Elsevier Science B.V.

[4] Ernest E. Onuiri, Oludele Awodele and Sunday A. Idowu (2014). Framework for Knowledge-Based Intelligent Clinical Decision support to Predict Comorbidity. International Journal of Advanced Research in Artificial Intelligence, (IJARAI) 3(4).

[5] Fulton M. M and Allen E.R(2005). Poly-pharmacy in elderly: a literature review. J Am Acad Nurse Pract; 17(4):123-32.
[6] Gunaratnam M. (2013). A Web-Based Perinatal Decision Support System Framework Using a Knowledge-BasedApproach to Estimate Clinical Outcomes: Neonatal Mortality and Preterm Birth in Twins Pregnancies. http://www.sce

[7] Hajjar E.R, Cafiero A.C, and Hanlon J.T (2007). Polypharmacy in elderly patients.;Am J Geriatr Pharmacother ;5(4):345-51.

[8] Hilmer S.N, Gnjidic D.(2009). The effects of polypharmacy in older adults. Clin Pharmacol Ther;85(1):86-98.

[9] Jakovljevic Mitro and Ljerka Ostojic (2013).Comorbidity and multimorbidity in medicine today. Challenges and opportunities for bringing separated branches of medicine closer to each other. Medicinia Academica Mostrariensia, 1(1).

[10] Linjakumpu T, Hartikainen S, Klaukka T, Veijola J, Kivela S.L and Isoaho R(2002). Use of medications and polypharmacy are increasing among the elderly. J Clin Epidemiol; 55(8):809-17.

[11] Mangoni A.A and Jackson S.H.D(2004). Age-related changes in pharmacokinetics and pharmacodynamics: basic principles and practical applications. $\mathrm{Br} \mathrm{J}$ Clin Pharmacol; 57(1):6-14.

[12] Paolo Fraaccarro, Mercedes Arguello Casteleiro, John Ainsworth, Iain Buchan (2015). Adoption of clinical decision support in multimorbidity: a systemic review. JMIR medical Informatics 3 (1)

[13] Power, D.J. (1999). Decision Support Systems Glossary.http://DSSResources.COM/glossary.

[14] Spinewine A, Schmader K.E, Barber N, Hughes C, Lapane K.L, Swine C, et al.(2007) Appropriate prescribing in elderly people: how well can it be measured and optimised? Lancet; 370(9582):173-84.

[15] Van den Akker M, Buntinx F, Metsemakers JFM, et al.(1998) .Multimorbidity in general practice: prevalence, incidence, and determinants of co-occurring chronic and recurrent diseases. J Clin Epidemiol. 1998; 51:367-375.

[16] Veehof L.J.G, Meyboom-de Jong B and HaaijerRaskamp F.M(2000). Poly-pharmacy in the elderly - a literature review. Eur J Gen Pract ;6(7):98-106.

[17] Von Lueder Thomas G. and Dan Atar (2014). Comorbidities and Polypharmacy Article in Heart Failure Clinics · April 2014 